\title{
Выращивание методом МОС-гидридной эпитаксии субмонослойных квантовых точек InGaAs/GaAs для возбуждения поверхностных плазмон-поляритонов
}

\author{
(C) Н.В. Байдусь ${ }^{1}$, В.А. Кукушкин ${ }^{2,3, \uparrow, ~ С . М . ~ Н е к о р к и н ~}{ }^{1}$, А.В. Круглов ${ }^{1,3}$, Д.Г. Реунов ${ }^{3}$ \\ ${ }^{1}$ Научно-исследовательский фризико-технический институт \\ Нижегородского государственного университета им. Н.И. Лобачевского, \\ 603950 Нижний Новгород, Россия \\ ${ }^{2}$ Институт прикладной физики Российской академии наук, \\ 603950 Нижний Новгород, Россия \\ ${ }^{3}$ Нижегородский государственный университет им. Н.И. Лобачевского, \\ 603950 Нижний Новгород, Россия \\ 『 E-mail: vakuk@appl.sci-nnov.ru
}

(Получена 15 октября 2018 г. Принята к печати 22 октября 2018 г.)

\begin{abstract}
Исследованы свойства квантовых точек InGaAs/GaAs, выращенных методом МОС-гидридной миграционно-стимулированной эпитаксии при пониженном давлении с применением субмонослойного осаждения. Длина волны их фотолюминесценции при $300 \mathrm{~K}$ находится в диапазоне $1.28-1.31$ мкм, ею можно управлять путем изменения температуры роста и числа циклов осаждения квантовых точек. Максимальная поверхностная плотность квантовых точек $3 \cdot 10^{10} \mathrm{~cm}^{-2}$. Выращены структуры с 1-3 слоями квантовых точек с толщинами спейсерных слоев между ними 5-12нм и селективным легированием последних (а также покровных слоев) углеродом (акцептор). Установлено, что фотолюминесценция квантовых точек характеризуется повышенной степенью поляризации в ортогональном плоскости структуры направлении, что должно благоприятствовать их применению для возбуждения поверхностных плазмон-поляритонов в светоизлучающих диодах Шоттки.
\end{abstract}

DOI: 10.21883/FTP.2019.03.47286.8999

\section{1. Введение}

Поверхностные плазмон-поляритоны представляют собой локализованные на границе раздела двух сред с различными знаками действительных частей их диэлектрических проницаемостей (обычно металла и полупроводника) самосогласованные колебания поляризации и электромагнитного поля [1]. Масштабы распределения последнего в них по всем трем координатам могут быть значительно меньше длины его волны как в вакууме, так и в любой из этих сред, взятых по отдельности. Это позволяет использовать электромагнитное поле оптического и инфракрасного частотных диапазонов (характеризующихся большим числом доступных источников) в микрооптоэлектронике, нанофотонике, микроскопии, биодетектировании и других приложениях.

Интересным объектом для исследования в качестве активной области плазмон-поляритонных источников являются квантовые точки (КТ) благодаря их высокому ограничивающему потенциалу и, как следствие, способности сохранять высокую интенсивность люминесценции при комнатной температуре [2].

В настоящей работе мы приводим результаты исследования гетероструктур с несколькими слоями КТ $\mathrm{InGaAs} / \mathrm{GaAs}$, адаптированных для плазмон-поляритонных светоизлучающих диодов Шоттки. Нашей целью была разработка способов повышения поверхностной плотности КТ в слое, снижения его дефектности, увеличения интенсивности фотолюминесценции (ФЛ) КТ и степени ортогональной плоскости структуры ее поляризации. Последняя особенно важна при использовании КТ как активных центров для возбуждения поверхностных плазмон-поляритонов на границе полупроводника с металлом. Это связано с тем, что чем больше степень ортогональной плоскости структуры поляризации ФЛ КТ, тем больше отношение ортогональной плоскости структуры компоненты дипольного момента перехода КТ по сравнению с его компонентой, лежащей в этой плоскости. В то же время известно [3], что поверхностные плазмон-поляритоны обладают в $\sim 2$ раза большей проекцией вектора электрического поля на ортогональное плоскости структуры направление, чем на эту плоскость. Поэтому эффективность их возбуждения КТ резко возрастает при увеличении ортогональной плоскости структуры компоненты дипольного момента перехода КТ по сравнению с его компонентой, лежащей в этой плоскости, т.е. степени ортогональной плоскости структуры поляризации ФЛ КТ в отсутствие металла.

Поскольку поверхностные плазмон-поляритоны быстро затухают вследствие омической диссипации их энергии в металле, то, если не принять специальных мер, из структуры будет выходить излучение поверхностных плазмон-поляритонов, сгенерированных лишь на малых расстояниях от ее торцов (не превышающих длины их поглощения, которая в ближнем инфракрасном диапазоне $\sim 7$ мкм [3]) и превратившихся на этих торцах в обычные фотоны, свободно покидающие образец. Поэтому нашей целью было также развитие метода рассеяния 
поверхностных плазмон-поляритонов в обычные фотоны на всей площади границы металла и полупроводника на искусственно созданных на этой границе неоднородностях [3]. В данной работе приводятся результаты создания таких неоднородностей методом „жертвенных“ КТ.

\section{2. Методика эксперимента}

Гетероструктуры выращивались методом МОС-гидридной эпитаксии при пониженном давлении на установке AIX 200RF на подложках GaAs с точной кристаллографической ориентацией (100). Источниками элементов являлись триметилгаллий, триметилиндий, триметилалюминий и арсин. Сначала выращивался буферный слой GaAs толщиной 0.5 мкм, ограничивающий слой $\mathrm{AlGaAs}$ толщиной 0.5 мкм (для предотвращения миграции фотосгенерированных носителей заряда в подложку) и слой GaAs толщиной 0.3 мкм при температуре $650^{\circ} \mathrm{C}$. Затем температура снижалась до температуры роста КТ, которая для разных образцов варьировалась в интервале $480-520^{\circ} \mathrm{C}$. Число циклов осаждения одного слоя КТ менялось в пределах 7-10, в одном цикле последовательно осаждалось 0.25 монослоя $\mathrm{Ga}$ и 0.25 монослоя In, а затем в течение 5 с поверхность выдерживалась в потоке арсина [4].

Покровный слой GaAs толщиной до 30 нм для разных структур выращивался при температуре роста КТ (структуры типа $A$ ). При создании образцов с поверхностными КТ толщина покровного слоя составляла всего 2 нм, после его осаждения структура охлаждалась (структуры $B$ ). Для создания искусственных поверхностных неоднородностей, на которых поверхностные плазмон-поляритоны могли бы рассеиваться в обычные фотоны, свободно покидающие структуру [3], нами были выращены образцы с ямками на поверхности (структуры типа $C$ ). Последние были созданы методом поверхностного слоя „жертвенных“ КТ: толщина покровного слоя делалась соизмеримой с высотой этих точек (6-8 нм), по окончании его осаждения подача прекурсоров прекращалась, а температура образца повышалась до $580^{\circ} \mathrm{C}$. После отжига в течение 100 с для растворения „жертвенных“ КТ и испарения индия нагрев выключался. Выращивались и комбинированные структуры типа $A B$, в которых одновременно присутствовали $1-2$ слоя объемных КТ и поверхностные КТ с толщиной спейсерного слоя между ними 6-12 нм. Достоинство таких структур в том, что они одновременно подходят для исследований ФЛ КТ и характеризации их параметров методом атомно-силовой микроскопии (АСМ). Для плазмон-поляритонных применений выращивались гетероструктуры типа $A C$ с объемными слоями КТ и поверхностным слоем ,жертвенных“ КТ с расстоянием между слоями 12 нм. В ряде случаев для повышения интенсивности ФЛ между слоями КТ и в покровном слое помещались $\delta$-слои углерода (акцептора) с поверхностной концентрацией $\sim 10^{12} \mathrm{~cm}^{-2}$.
Исследование образцов с самоорганизованными КТ InGaAs/GaAs было выполнено на ACM Solver PRO-M („NT-MDT“, Россия) в полуконтактном режиме на воздухе. Использовались зонды NT-MDT ETALON HA_HR. Радиус закругления кончика зонда составлял не более 10 нм (по данным производителя). Для образцов с поверхностными КТ была выполнена обработка и численная характеризация полученных АСМ-изображений с целью нахождения геометрических размеров и параметров массивов саморганизованных КТ. Был проведен анализ АСМ-изображений размером $1 \times 1$ мкм и определены высоты КТ, их латеральные размеры и поверхностная концентрация, построены гистограммы распределения двух первых указанных параметров.

Гетероструктуры тестировались на спектрометре RPM 2000, позволяющем измерять спектры ФЛ в диапазоне длин волн 600-1600 нм и карту ФЛ при $300 \mathrm{~K}$ при фотовозбуждении лазерами с длиной волны 780 и 530 нм. Спектральные и поляризационные зависимости ФЛ при температурах 77 и $300 \mathrm{~K}$ регистрировались с использованием монохроматора МДР-23 и германиевого детектора с применением синхронного детектирования сигнала. Измерения линейной поляризации излучения со сколотых граней структуры производились с помощью призмы Глана-Тейлора.

\section{3. Экспериментальные результаты и их обсуждение}

На рис. 1, $а$ представлено АСМ-изображение структуры А1422 размером $1 \times 1$ мкм с поверхностными КТ, выращенными при температуре $520^{\circ} \mathrm{C}$. Наблюдается сравнительно однородный массив КТ с поверхностной концентрацией $8 \cdot 10^{9} \mathrm{~cm}^{-2}$. На образце А1422 концентрация крупных дислоцированных кластеров (ДК) почти на 2 порядка меньше, чем псевдоморфных островков (ни один из них не попадает в кадр $1 \times 1$ мкм, их поверхностная концентрация составляет около $10^{8} \mathrm{~cm}^{-2}$ ). Проявляется высокая однородность псевдоморфных островков (которые являются КТ) как по латеральному размеру, так и по высоте. Средний латеральный размер КТ составил $66 \pm 14$ нм, что (с учетом эффекта конволюции) позволяет оценить истинный размер КТ величиной 54 нм. На гистограмме распределения по высоте (см. вставку) видно, что КТ на образце А1422 имеют бимодальное распределение по высотам с максимумами при 3 и 9 нм.

Для повышения поверхностной концентрации КТ были проведены опыты по их выращиванию при пониженной температуре, вариации числа циклов осаждения и увеличению числа слоев КТ. На рис. $1, b$ представлены АСМ-изображения поверхности структуры типа $A B$ с КТ, выращенными при пониженной температуре $480^{\circ} \mathrm{C}$. Структура содержит 3 слоя точек с толщиной спейсеров между ними 12 нм. Последний слой - поверхностный. Вследствие понижения температуры формирования КТ их средняя высота уменьшилась до 4-5 нм и значительно возросли их поверхностная концентрация (до 


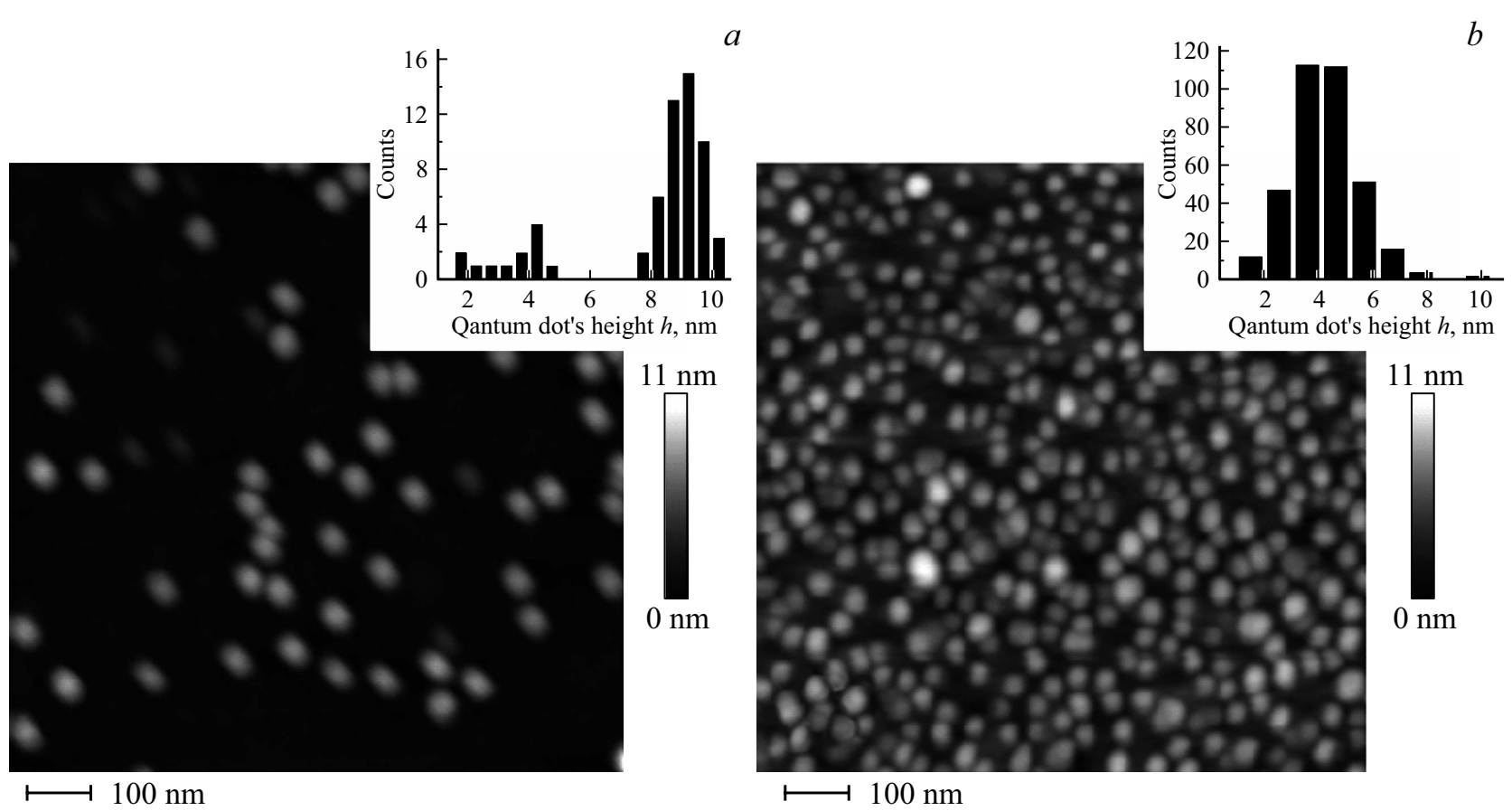

Рис. 1. АСМ-изображения поверхности и гистограммы распределения по высотам (на вставках) KT InGaAs/GaAs, сформированных при $520(a)$ и $480^{\circ} \mathrm{C}(b)$.

$\left.3.5 \cdot 10^{10} \mathrm{~cm}^{-2}\right)$ и дисперсия по высоте, что можно наблюдать на построенной гистограмме (см. вставку на рис. $1, b)$.

На рис. 2 кривая 1 показывает спектр ФЛ КТ (структура типа $A$ ), выращенных при $520^{\circ} \mathrm{C}$ при 10 циклах осаждения с покровным слоем 30 нм на точно ориентированной (100) подложке. Наблюдаются два сравнительно узких пика спектра ФЛ, связанных с оптическими

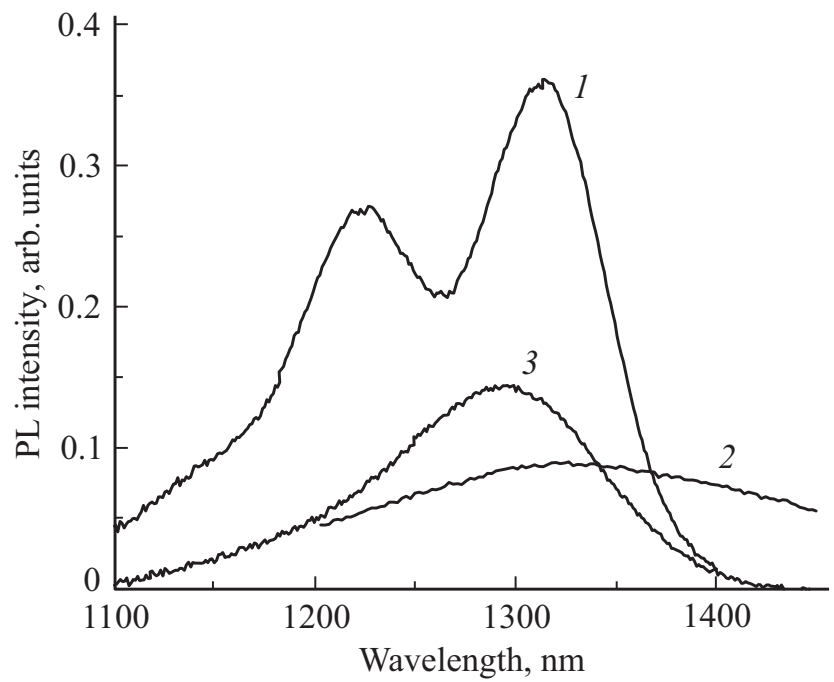

Рис. 2. Спектры ФЛ КТ: 1 - один слой КТ в объеме структуры (температура формирования КТ $520^{\circ} \mathrm{C}$ ), 2 - три слоя КТ в объеме структуры, выращенных при $480^{\circ} \mathrm{C}, 3$ - три слоя КТ в объеме структуры с ямками на ее поверхности (температура роста $490^{\circ} \mathrm{C}$ ). переходами с участием основных и возбужденных состояний. Длина волны основного перехода больше 1.3 мкм. Кривая 2 показывает спектральную зависимость ФЛ структуры с тремя слоями КТ, выращенной при $480^{\circ} \mathrm{C}$. Из сравнения этих спектров видно, что положение максимума ФЛ КТ при понижении температуры роста остается в области 1.3 мкм, но ширина спектра значительно увеличивается. Последнее, вероятно, связано как с увеличением дисперсии размеров КТ, что согласуется с данными АСМ-измерений, так и с различием размеров КТ в слоях.

На рис. 3 приведены АСМ-изображения структуры, в которой имеются три слоя КТ, выращенных при $490^{\circ} \mathrm{C}$ на точно ориентированной подложке (100), и ямки на поверхности, образовавшиеся после стравливания „жертвенного“ слоя КТ. Пониженная температура роста КТ и уменьшенное число циклов осаждения позволили избежать появления больших дислоцированных островков, поэтому ямки имеют размер, соизмеримый с размером КТ, показанных на рис. $1, b$. Спектры ФЛ этих КТ приведены на рис. 2 (кривая 3). Видно, что наличие слоя стравленных КТ на поверхности структуры не приводит к ухудшению люминесцентных свойств КТ в объеме структуры, а интенсивность и ширина спектра их ФЛ определяются температурой их формирования.

В заключение были выполнены поляризационные измерения ФЛ КТ. На рис. 4 для примера показаны спектральные зависимости ФЛ КТ с различными поляризациями для одной из структур с ямками на поверхности. Излучение выводилось со сколотого края образца. Видно, что для данной структуры ФЛ с ортогональной поверхности структуры поляризацией интенсивнее, 
Параметры структур (длина волны максимума спектра ФЛ $\lambda_{\max }$, толщина покровного слоя $d_{\text {сар }}$, наличие легирования углеродом, состояние поверхности, $I_{\perp} / I_{\|}$и вычисленное по нему отношение модуля ортогональной плоскости структуры составляющей дипольного момента излучающего перехода в КТ к модулю его составляющей, параллельной этой плоскости, $\left|d_{y}\right|^{2} /\left|d_{\|}\right|^{2}$, см. разд. 4)

\begin{tabular}{c|l|c|c|c|c|c|c}
\hline $\begin{array}{c}\text { № } \\
\text { образца }\end{array}$ & $\begin{array}{c}\lambda_{\max } \\
\text { мкм }\end{array}$ & $\begin{array}{c}d_{\text {сар }}, \\
\text { нм }\end{array}$ & $\begin{array}{c}\text { Наличие } \\
\text { легирования }\end{array}$ & $\begin{array}{c}\text { Состояние } \\
\text { поверхности }\end{array}$ & $\begin{array}{c}\text { Число слоев } \\
\text { КТ в объеме }\end{array}$ & $I_{\perp} / I_{\|}$ & $\left|d_{y}\right|^{2} /\left|d_{\|}\right|^{2}$ \\
\hline А1426 & 1.29 & 30 & Есть & Гладкая & 1 & 1.75 & 0.94 \\
A1462 & 1.3 & 2 & $\gg$ & Поверхностные КТ & 2 & 1.15 & 0.59 \\
A1465 & 1.26 & 10 & Ямки & 3 & 1.6 & 0.85 \\
А1447 & 1.29 & 12 & Нет & $\gg$ & 2 & 0.84 & 0.4
\end{tabular}

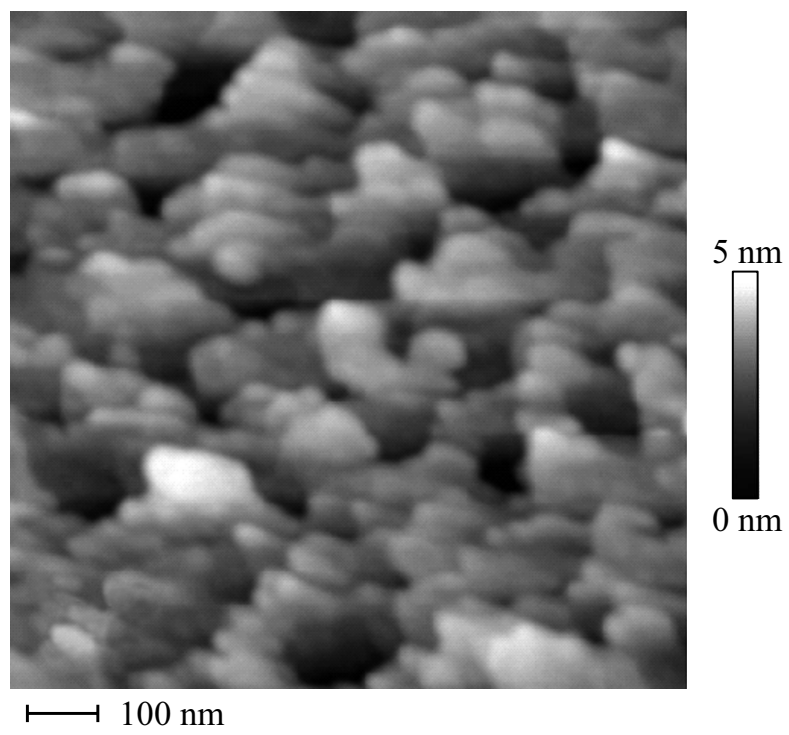

Рис. 3. АСМ-снимки поверхности структуры с тремя слоями КТ, выращенными на точно ориентированной подложке (100), и ямками на поверхности, образовавшимися после стравливания „жертвенного“ слоя КТ.

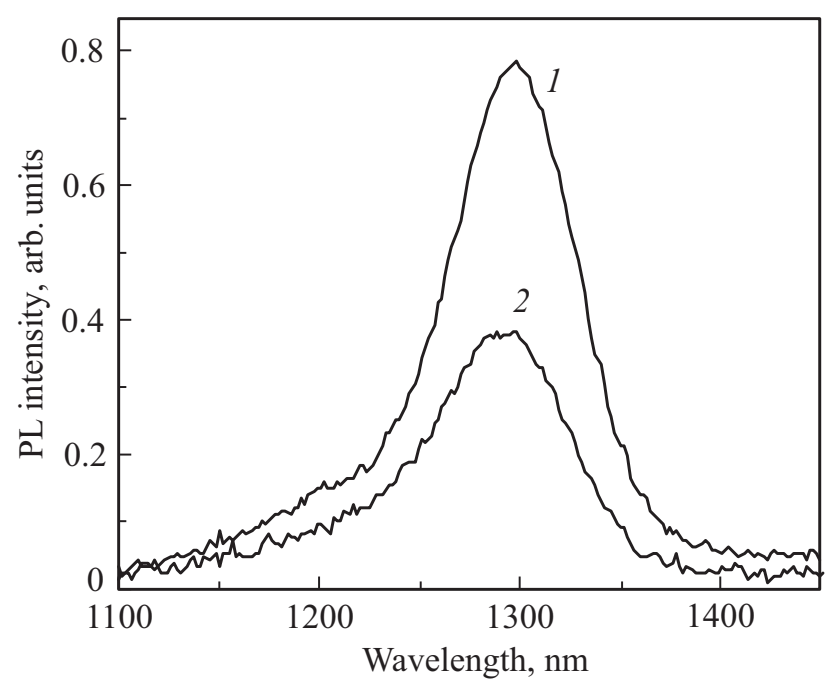

Рис. 4. Спектры ФЛ КТ с различными поляризациями, измеренные со сколотого края образца: 1 - ортогональная, 2 - параллельная плоскости структуры поляризации. чем с лежащей в плоскости структуры. Отношения соответствующих интенсивностей ФЛ в максимумах их спектров, $I_{\perp} / I_{\|}$, для различных структур приведены в таблице.

Анализ данных, приведенных в таблице, позволяет сделать вывод о существенном повышении $I_{\perp} / I_{\|}$при легировании углеродом области вокруг слоя КТ. Это согласуется с результатами работы [5] о влиянии легирования акцепторами на величину $I_{\perp} / I_{\|}$и с выводами нашей предыдущей работы [3] о желательности заполнения подзоны легких дырок в GaAs, в основном ответственных за ортогональную плоскости структуры поляризацию ФЛ КТ за счет непрямых в пространстве переходов в сильном электрическом поле. Наличие последнего в нашем случае обеспечивается расположением КТ в области пространственного заряда вблизи поверхности структуры. Из таблицы видно, что $I_{\perp} / I_{\|}$возрастает при увеличении высоты КТ: структура А1426 была выращена при $520^{\circ} \mathrm{C}$ и, согласно рис. 1 и таблице, высота КТ и $I_{\perp} / I_{\|}$для нее больше, чем для структуры А1462, выращенной при $480^{\circ} \mathrm{C}$. Подобный эффект наблюдался ранее [6-12], где эффективная высота КТ возрастала за счет увеличения числа их слоев и туннельной связи вдоль оси роста КТ в различных слоях, а также в [13] для высоких („колоннообразных“) КТ. Следует заметить, что преобладание в люминесценции КТ перпендикулярной к плоскости структуры поляризации встречается редко [14] и для контролируемого применения требует дальнейшего исследования.

\section{4. Расчет $\left|d_{y}\right|^{2} /\left|d_{\|}\right|^{2}$ по экспериментальным значениям $I_{\perp} / I_{\|}$}

Зная $I_{\perp} / I_{\|}$, можно найти отношение модуля ортогональной плоскости структуры составляющей дипольного момента излучающего перехода в КТ к модулю его составляющей, параллельной этой плоскости, $\left|d_{y}\right|^{2} /\left|d_{\|}\right|^{2}$. Для этого введем декартову систему координат с началом в рассматриваемой КТ, ортогональной плоскости структуры осью $y$ и лежащими в этой плоскости осями $x$ и $z$, причем последняя направлена от КТ к наблюдателю (см. рис. 5). Дипольный момент излучающего 


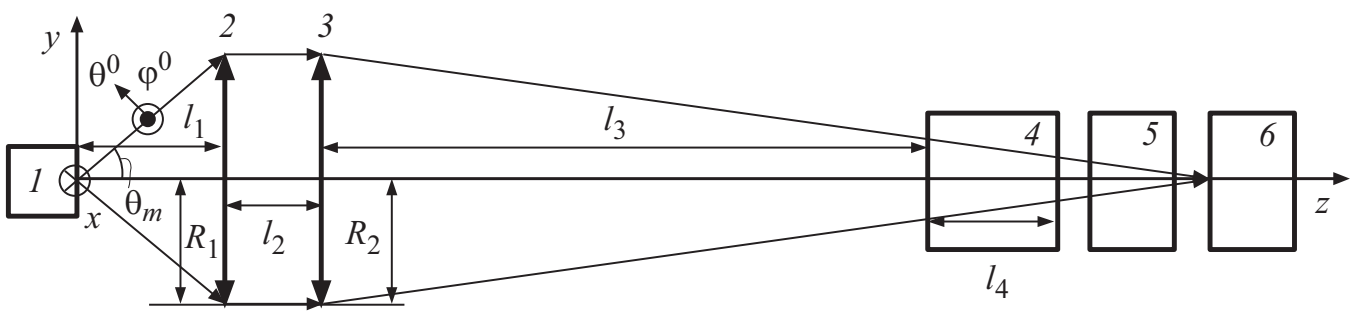

Рис. 5. Схема поляризационных измерений излучения КТ и используемая система координат: $1-$ образец, $2-$ первая линза конденсора, 3 - вторая линза конденсора, $4-$ призма Глана (поляризатор), $5-$ фильтр, $6-$ монохроматор, $l_{1}=4 \mathrm{~cm}, R_{1}=4$ см, $l_{2}=4 \mathrm{~cm}, R_{2}=4 \mathrm{~cm}, l_{3}=13 \mathrm{~cm}, l_{4}=5 \mathrm{~cm}$.

перехода КТ d имеет координаты $\left(d_{x}, d_{y}, d_{z}\right)$. Пусть $\mathbf{r}=r(\sin \theta \cos \varphi, \sin \theta \sin \varphi, \cos \theta)$ - радиус-вектор, проведенный из КТ к точке первой линзы конденсора, имеющей полярную и азимутальную угловые координаты $\theta$ и $\varphi$ в сферической системе координат с осью $z$, a $\mathbf{r}^{0}$ - направленный вдоль него единичный вектор. Координатам $\theta$ и $\varphi$ соответствуют ортогональные $\mathbf{r}^{0}$ единичные орты $\boldsymbol{\theta}^{0}$ и $\varphi^{0}$ (причем последний лежит в плоскости $x y$ ). Как хорошо известно [15], при дипольном излучении электрическое поле $\mathbf{E}$ в волновой зоне пропорционально $\left[\left[\mathbf{d r}^{0}\right] / \mathbf{r}^{0}\right] / r \equiv \mathbf{r}^{0}\left(\mathbf{r}^{0} \mathbf{d}\right) / r-\mathbf{d} / r$, т. е. ортогонально $\mathbf{r}^{0}$ и поэтому может быть представлено в виде $\mathbf{E}=E_{\theta} \boldsymbol{\theta}^{0}+E_{\varphi} \varphi^{0}$, где

$$
\begin{aligned}
& E_{\theta} \propto\left(\boldsymbol{\theta}^{0}\left[\left[\mathbf{d} \mathbf{r}^{0}\right] \mathbf{r}^{0}\right]\right) / r=-\left(\theta^{0} \mathbf{d}\right) / r, \\
& E_{\varphi} \propto\left(\varphi^{0}\left[\left[\mathbf{d} \mathbf{r}^{0}\right] \mathbf{r}^{0}\right]\right) / r=-\left(\varphi^{0} \mathbf{d}\right) / r .
\end{aligned}
$$

При преломлении в первой линзе конденсора расходящийся пучок становится параллельным. При этом, очевидно, компонента $\mathbf{E}$, направленная вдоль $\boldsymbol{\theta}^{0}$, поворачивается на угол $\theta$ вокруг $\varphi^{0}$ в направлении к наблюдателю, так что она оказывается лежащей в плоскости $x y$, а компонента вдоль $\varphi^{0}$ направление не меняет. Таким образом, E оказывается лежащим в плоскости $x y$. Одновременно происходит пропорциональное $\sqrt{\cos \theta}$ уменьшение величин обеих компонент $\mathbf{E}$, поскольку площадь волнового фронта $r^{2} \sin \theta d \theta d \varphi$, соответствующая пучку лучей, распространяющихся от КТ в элементе телесного угла $\sin \theta d \theta d \varphi$, после преломления в первой линзе конденсора становится равной $r^{2} \sin \theta d \theta d \varphi / \cos \theta$, а потоки энергии через эти площади должны быть равны. Таким образом, на выходе первой линзы конденсора имеем

$$
E_{x} \propto \sqrt{\cos \theta}\left[\left(\mathbf{d} \varphi^{0}\right) \sin \varphi-\left(\mathbf{d} \boldsymbol{\theta}^{0}\right) \cos \varphi\right] / r
$$

и

$$
E_{y} \propto-\sqrt{\cos \theta}\left[\left(\mathbf{d} \varphi^{0}\right) \cos \varphi+\left(\mathbf{d} \boldsymbol{\theta}^{0}\right) \sin \varphi\right] / r .
$$

Соответствующая мощность излучения с ортогональной плоскости структуры поляризацией пропорциональна

$$
\int_{0}^{\theta_{m}} \int_{0}^{2 \pi}\left|E_{y}\right|^{2} r^{2} \sin \theta d \theta d \varphi / \cos \theta
$$

a с параллельной -

$$
\int_{0}^{\theta_{m}} \int_{0}^{2 \pi}\left|E_{x}\right|^{2} r^{2} \sin \theta d \theta d \varphi / \cos \theta,
$$

где интегрирование выполняется по телесному углу, под которым из КТ видна первая линза конденсора, $\theta_{m}$ соответствующий максимальный угол $\theta, \operatorname{tg} \theta_{m}=R_{1} / l_{1}$.

Поскольку $l_{3}+l_{4} \gg R_{2}$, после второй линзы конденсора пучок сходится под малым углом, т.е. остается почти параллельным. Поэтому на призме Глана (поляризаторе) мощности излучения с ортогональной плоскости структуры и лежащей в ней поляризациями остаются примерно такими же, как на выходе первой линзы конденсора. Далее, поскольку размеры образца много меньше расстояний от него до первой линзы конденсора, можно считать, что все КТ находятся в одном и том же месте - начале выбранной выше декартовой системы координат. Их излучение является спонтанным и поэтому должно складываться некогерентно. В силу однородности всех характеристик структуры в ее плоскости $x z$ средние по КТ $\left|d_{x}\right|^{2}$ и $\left|d_{z}\right|^{2}$ должны быть равны друг другу. Обозначим их как $\left|d_{\|}\right|^{2} / 2$, где $\left|d_{\|}\right|^{2}-$ характерный квадрат модуля параллельной плоскости структуры компоненты дипольного момента излучающего перехода КТ. Для среднего по КТ $\left|d_{y}\right|^{2}$ сохраним прежнее обозначение. Выполняя интегрирование, в итоге получим для измеряемого

$$
\frac{I_{\perp}}{I_{\|}}=\frac{\int_{0}^{\theta_{m}} \int_{0}^{2 \pi}\left|E_{y}\right|^{2} r^{2} \sin \theta d \theta d \varphi / \cos \theta}{\int_{0}^{\theta_{m}} \int_{0}^{2 \pi}\left|E_{x}\right|^{2} r^{2} \sin \theta d \theta d \varphi / \cos \theta}
$$

формулу

$$
\begin{aligned}
& \left|d_{y}\right|^{2}\left(5-3 \cos \theta_{m}-\cos ^{2} \theta_{m}-\cos ^{3} \theta_{m}\right)+ \\
& \frac{I_{\perp}}{I_{\|}}=\frac{+\left|d_{\|}\right|^{2}\left(3-5 \cos \theta_{m}+\cos ^{2} \theta_{m}+\cos ^{3} \theta_{m}\right) / 2}{\left|d_{y}\right|^{2}\left(1-\cos \theta_{m}\right)^{3} / 3+} \text {. } \\
& +\left|d_{\|}\right|^{2}\left[23 / 3-7 \cos \theta_{m}-\cos ^{2} \theta_{m}+\cos ^{3}\left(\theta_{m}\right) / 3\right] / 2
\end{aligned}
$$

Из нее, зная экспериментальное отношение $I_{\perp} / I_{\|}$ и $\theta_{m}$, можно вычислить $\left|d_{y}\right|^{2} /\left|d_{\|}\right|^{2}$. Результаты такого 
вычисления приведены в последнем столбце таблицы. Из нее видно, что у КТ с наибольшим $I_{\perp} / I_{\|}$величина ортогональной плоскости структуры компоненты дипольного момента излучающего перехода близка к величине его компоненты параллельной плоскости структуры, т.е. такие КТ способны эффективно возбуждать поверхностные плазмон-поляритоны.

\section{5. Заключение}

Таким образом, разработана технология выращивания адаптированных для возбуждения поверхностных плазмон-поляритонов структур с квантовыми точками $\mathrm{InGaAs/GaAs.} \mathrm{Она} \mathrm{основана} \mathrm{на} \mathrm{методе} \mathrm{MOC-гидридной}$ миграционно-стимулированной эпитаксии при пониженном давлении с применением субмонослойного осаждения. Выращены образцы с несколькими слоями квантовых точек в объеме структуры и ямками на ее поверхности. Последние созданы путем испарения слоя поверхностных ,жертвенных“ квантовых точек и после заполнения их металлом будут являться центрами рассеяния поверхностных плазмон-поляритонов в свободно покидающие структуру обычные фотоны. Установлено, что фотолюминесценция квантовых точек характеризуется повышенной степенью поляризации в ортогональном плоскости структуры направлении, на основе чего с помощью расчетов показано, что дипольный момент их излучающего перехода имеет примерно равные проекции на это направление и плоскость структуры. Последнее позволет ожидать, что выращенные квантовые точки будут эффективно возбуждать поверхностные плазмонполяритоны в светоизлучающих диодах Шоттки.

Работа выполнена при финансовой поддержке РФФИ (грант 16-02-00450-а) с использованием приборной базы Центра коллективного пользования Научнообразовательного центра „Физика твердотельных наноструктур“ Нижегородского государственного университета им. Н.И. Лобачевского.

\section{Список литературы}

[1] С.А. Майер. Плазмоника: теория и приложения (М., Ижевск, R\&C Dynamics, 2011) гл. 2. [S.A. Maier. Plasmonics: Fundamentals and Applications (Springer Verlag, Berlin, 2007) chap. 2].

[2] A. Chahboun, M.I. Vasilevskiy, N.V. Baidus, A. Cavaco, N.A. Sobolev, M.C. Carmo, E. Alves, B.N. Zvonkov. J. Appl. Phys., 103 (8), 083548 (2008).

[3] Н.В. Байдусь, В.А. Кукушкин, Б.Н. Звонков, С.М. Некоркин. ФТП, 50 (11), 1576 (2016).

[4] D.L. Huffaker, D.G. Deppe. Appl. Phys. Lett., 73 (4), 520 (1998).

[5] K.Y. Chuang, C.Y. Chen, T.E. Tzeng, J.Y. Feng, T.S. Lay. Physica E, 40 (6), 1882 (2008).

[6] P. Ridha, L. Li, A. Fiore, G. Patriarche, M. Mexis, P.M. Smowton. Appl. Phys. Lett., 91 (19), 191123 (2007).
[7] L.H. Li, M. Mexis, P. Ridha, M. Bozkurt, G. Patriarche, P.M. Smowton, P. Blood, P.M. Koenraad, A. Fiore. Appl. Phys. Lett., 95 (22), 221116 (2009).

[8] M. Usman. J. Appl. Phys., 110 (9), 094512 (2011).

[9] M. Usman, S. Heck, E. Clarke, P. Spencer, H. Ryu, R. Murray, G. Klimeck. J. Appl. Phys., 109 (10), 104510 (2011).

[10] M. Usman, T. Inoue, Y. Harda, G. Klimeck, T. Kita. Phys. Rev. B, 84 (11), 115321 (2011).

[11] Y. Ikeuchi, T. Inoue, M. Asada, Y. Harada, T. Kita, E. Taguchi, H. Yasuda. Appl. Phys. Express, 4 (6), 062001 (2011).

[12] М.М. Соболев, И.М. Гаджиев, И.О. Бакшаев, В.Н. Неведомский, М.С. Буяло, Ю.М. Задиранов, Р.В. Золотарева, Е.Л. Портной. ФТП, 46 (1), 96 (2012).

[13] P. Ridha, L. Li, M. Rossetti, G. Patriarche, A. Fiore. Optical Quant. Electron., 40 (2-4), 239 (2008).

[14] P. Jayavel, H. Tanaka, T. Kita, O. Wada, H. Ebe, M. Sugawara, J. Tatebayashi, Y. Arakawa, Y. Nakata, T. Akiyama. Appl. Phys. Lett., 84 (11), 1820 (2004).

[15] Л.Д. Ландау, Е.М. Лифшиц. Теория поля (М., Наука, 1988). [L.D. Landau, E.M. Lifshitz. The Classical Theory of Fields (Oxford, Butterworth-Heinenann, 1996)].

Редактор Г.А. Оганесян

\section{Metalorganic Chemical Vapor Deposition Epitaxy Growth of Submonolayer Quantum Dots InGaAs/GaAs for the Excitation of Surface Plasmon-Polaritons}

\author{
N.V. Baidus ${ }^{1}$, V.A. Kukushkin ${ }^{2,3}$, S.M. Nekorkin ${ }^{1}$, \\ A.V. Kruglov ${ }^{1,3}$, D.G. Reunov ${ }^{3}$ \\ ${ }^{1}$ Research Physico-Technical Institute \\ of the Nizhny Novgorod State University \\ named after N.I. Lobachevsky, \\ 603950 Nizhny Novgorod, Russia \\ 2 Institute of Applied Physics \\ of the Russian Academy of Science, \\ 603950 Nizhny Novgorod, Russia \\ ${ }^{3}$ Nizhny Novgorod State University \\ named after N.I. Lobachevsky, \\ 603950 Nizhny Novgorod, Russia
}

